\title{
Pathological response post neoadjuvant therapy for locally advanced rectal cancer is an independent predictor of survival.
}

\author{
ON, J., SHIM, J., MACKAY, C., MURRAY, G., SAMUEL, L., PARNABY, C. \\ and RAMSAY, G.
}

This is the peer reviewed version of the following article: ON, J., SHIM, J., MACKAY, C., MURRAY, G., SAMUEL, L., PARNABY, C. and RAMSAY, G. 2021. Pathological response post neoadjuvant therapy for locally advanced rectal cancer is an independent predictor of survival. Colorectal disease [online], 23(6), pages 1326-1333, which has been published in final form at https://doi.org/10.1111/codi.15512. This article may be used for

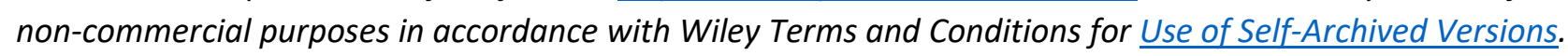




\title{
Pathological response post neoadjuvant therapy for locally advanced rectal cancer is an independent predictor of survival
}

\author{
Jason On ${ }^{1}$, Joanna Shim ${ }^{2}$, Craig Mackay ${ }^{1}$, Graeme Murray ${ }^{3}$, Leslie Samuel ${ }^{4}$, Craig Parnaby ${ }^{1}$, \\ George Ramsay ${ }^{1,5}$ \\ Department of Colorectal Surgery, Aberdeen Royal Infirmary, NHS Grampian ${ }^{1}$ \\ Department of Epidemiology, University of Aberdeen ${ }^{2}$ \\ Pathology, Division of Applied Medicine, School of Medicine and Dentistry, University of \\ Aberdeen $^{3}$ \\ Department of Clinical Oncology, Aberdeen Royal Infirmary, NHS Grampian ${ }^{4}$ \\ Rowett Institute for Nutrition and Health, University of Aberdeen ${ }^{5}$
}

Corresponding author:

Jason On BM, FRCS

ST8 General and Colorectal Surgery

Laparoscopic Colorectal Surgery \& Training Unit - Aberdeen Royal Infirmary

Foresterhill - Aberdeen - AB25 2ZN - UK

+44 (0)7788988427

wei.on@nhs.scot

Keywords: Rectal Cancer, tumour regression grade, pathological response

Short Title: Pathological response in rectal cancer

Disclaimers: None

Sources of finance: None

Abstract word count: 226

Text word count: 3101

References count: 27

No. of Tables: 3

No. of Figures: 2 


\section{Additional Information}

Ethics approval and consent to participate:

- This is an assessment of clinical datasets and this was approved by the regional Caldicott guardian.

- The study was performed in accordance with the declaration of Helsinki.

Consent for publication:

- This is not applicable as there is no identifiable individual patient's data

Data availability:

- The data that supports this study are available on request from the corresponding author $(\mathrm{JO})$ upon reasonable request.

Conflict of interest:

- There is no conflict of interest.

Funding:

- No funding disclosure.

Authors' contribution:

- JO collected the evidence and wrote the final manuscript. JS performed the data and statistical analysis. CM, GM and GR contributed to the database used in this study. GM, LS, CP and GR guided the writing of the manuscript. All authors critically reviewed the manuscript for important intellectual content. 


\section{Abstract}

Background

Neoadjuvant treatment (NaT) for locally advanced rectal cancer prior to surgery has led to improved outcomes. However, the relationship between pathological response to NaT and survival is not entirely clear. The aim of this study was to assess the degree of pathological response to $\mathrm{NaT}$ on survival outcomes.

\section{Methods}

Clinical and pathological data were collected from a prospectively maintained pathology database between 2005 and 2017. The primary outcome was the overall survival based on pathological response categorized as complete, good partial, partial and minimal. Univariate and multivariate analysis were conducted to identify variables predictive of survival. Cox proportional hazard ratios were used for survival.

\section{$\underline{\text { Results }}$}

A total of 596 patients had surgery following $\mathrm{NaT}$ for locally advanced rectal cancer. The median follow-up was 4.57 years (IQR 2.21-8.15 years). The overall survival for complete pathological response was $75.6 \%$ versus $37.3 \%$ for minimal response ( $p$ $<0.001)$. The overall survival at the end of the study in the good partial versus partial response group was $58.9 \%$ versus $39 \%$, ( $p<0.001$ ). On multivariate analysis, the degree of pathological response remains an independent variable for overall and disease specific survival across all categories.

\section{Discussion}

In addition to other pathological variables, the degree of pathological response to $\mathrm{NaT}$ is an independent predictor for survival outcomes. Future verification of these findings elsewhere could support NaT response being used for adjuvant therapy decision making. 
What does this paper add to the literature?

Emphasis should be placed on tumour regression grade on outcomes. NaT response has previously been shown to be an independent predictor of survival. Our study has demonstrated further stratification of the tumour regression grade correlates with survival and we therefore advocate its regular reporting. 
Introduction

There has been much advancement in the management of rectal cancer over the last few decades. Previously, the prognosis for locally advanced rectal cancer was poorer compared to stage equivalent colonic malignancy (1). However, the introduction of total mesorectal excision (TME) and neoadjuvant treatment (NaT) has led to improved outcomes for patients with magnetic resonance imaging (MRI) defined high risk rectal cancer (1)(2). NaT before TME surgery is standard care for the management of locally advanced rectal cancer following several high profile studies reporting on its' benefits (3)(4). With modern management, patients with rectal cancer have longer survival compared to staged equivalent colon cancer (5).

A proportion of patients undergoing $\mathrm{NaT}$ achieved pathological complete response $(p C R)$ which is defined as the absence of any residual cancer cells in the resected pathological specimen. A recent study from the Netherlands has demonstrated a pCR rate of $17.5 \%$ and a good response rate of $22.6 \%$ in patients who had undergone NaT (6). However, response to $\mathrm{NaT}$ is unpredictable and there can be varying degrees of regression.

Assessment of the histopathological response is made by using a tumour regression grading (TRG) system. Mandard et al was the first to develop a 5 point TRG system to assess pathological response to treatment in oesophageal cancer (7). However, Mandard TRG system may not be suitable for grading response in rectal cancers due to the differences in tumour biology. An accurate TRG system is a necessity as clinical decisions are being made based on the pathological outcomes. At present, several other TRG systems for rectal cancer exists including the American Joint Committee on Cancer (AJCC), Dworak and Ryan systems (8).

Whilst complete response has been reported to have excellent outcomes (9)(10), it is unclear if survival can be predicted by the other grades of response to $\mathrm{NaT}$. If the tumour has been completely resected, any potential neoadjuvant response could be negated by TME surgical resection. However, the degree of pathological response to neoadjuvant treatment may nevertheless impact on the survival outcomes (overall and 
disease-specific) and hence an important consideration for both clinicians and patients $(11)(12)(13)$.

The purpose of the present study is to evaluate the impact of our tumour regression grading system on long term survival outcomes in patients who had undergone NaT for locally advanced rectal cancer. 
Methods

Study design

This is a single centre case series analysis of a prospectively collated dataset. All patients who fulfilled the inclusion criteria between January 2005 and October 2017 were included. Rectal cancer was defined as a presence of cancer $15 \mathrm{~cm}$ or less from the anal verge on sigmoidoscopy.

Inclusion \& exclusion criteria

All patients diagnosed with primary rectal cancer who underwent $\mathrm{NaT}$ followed by curative resection were included. Patients were excluded if rectal cancer resection was performed without $\mathrm{NaT}$, beyond TME, performed with palliative intent, for recurrent disease or delayed (e.g. regrowth from watch and wait programme).

\section{Treatment}

All patients were discussed in the multi-disciplinary (MDT) meeting, had colonoscopy, staging CT and MRI scan. Neoadjuvant treatment was administered if the tumour is locally advanced. This is defined on thin slice MRI imaging, the circumferential resection margin (CRM) was involved either with tumour or lymph nodes. Involved CRM was defined $\leq 1 \mathrm{~mm}$ from mesorectal fascia, levators or intersphincteric plane. Patients who were deemed fit were offered radiotherapy with concurrent capecitabine. The radiotherapy dose was $45 \mathrm{~Gy}$ in 25 fractions over 5 weeks. Surgery was performed 8 to 10 weeks following treatment. In the presence of extensive extramural venous invasion (EMVI), gross involvement of the CRM or lymph node involvement on MRI, oxaliplatin and capecitabine were selectively used in combination with radiotherapy (14). Patients with significant co-morbidities were treated with radiotherapy alone. A radiotherapy dose of $25 \mathrm{~Gy}$ in 5 fractions over 5 days was used, with surgery again performed 8 to 10 weeks following treatment. In cases where the volume of small bowel adjacent to the tumour (on the radiotherapy plane) or deemed at significant risk of persistent presence in the radiotherapy field, neoadjuvant chemotherapy alone was used. 
Patients underwent either an anterior resection or abdomino-perineal excision of rectum dependent on the distance of the tumour from the anal verge. All surgeries were performed with principles of TME.

\section{Pathological sampling and grading}

The resected specimens were sent directly to the laboratory fresh (without fixative). All specimens were opened along the anti-mesenteric border of the sigmoid colon and upper rectum to the level of the anterior peritoneal reflection, washed in cold water and fixed in formalin for at least 48 hours. The fixed specimens were serially sliced in the horizontal plane. Appropriate tissue blocks were taken for histo-pathological assessment. All specimens were processed and embedded when there was no visible macroscopic evidence of residual tumour. Mega (whole amount) blocks were used for embedding the slices of rectum. When there was macroscopic evidence of residual tumour all residual tumour were embedded.

The tumour regression response to neoadjuvant therapy was developed by an expert gastrointestinal pathologist (GM) and has been validated in a previous smaller study by the same pathology team (15). Brown et. al, 2016 assessed the relationship between histopathological response grade to neoadjuvant therapy and overall survival in the same cohort of patients from 2005 to 2011. The TRG system was based on the degree of fibrosis and inflammation compared to residual histologically viable tumour. These parameters were incorporated into a four points scale to give an overall assessment of response of tumour to $\mathrm{NaT}$ (complete, good partial, partial and minimal response).

The TRG system is similar to the current recommended reporting proforma for rectal cancer specimens by the Royal College of Pathologists UK (16) and has been used consistently throughout the study period. TNM 5 staging system was also used throughout the study period.

Changes over time 
A formal 'watch and wait' programme was launched in April 2017. Up until this point it was performed in an ad-hoc manner for patients either at very high risk for surgical intervention or those who refused surgery. Post neoadjuvant treatment MRI was performed routinely from 2013, before which post treatment MRI was used selectively. The bowel cancer screening programme started in our institution in June 2007.

Data analysis

Data were obtained from a prospectively maintained pathology registry. All records in this registry were anonymised. Patient demographics, histological diagnosis, lymph node ratio (LNR), presence of pathologically identified extramural venous invasion, quality of TME, CRM, final pathological staging and pathological response to NaT were collected. Survival data were obtained from the Information Services Division, Scotland.

\section{Outcomes}

The primary endpoint of our study was the overall survival, defined as time from completion of treatment to death of any cause. Disease specific survival, defined as time from completion of treatment to death attributed to the disease itself, is a secondary endpoint.

Subgroup analysis assessed the overall and disease specific survival in patients who had complete excision of their tumour (No R1 or R2 resections). A descriptive account of survival in patients with incomplete excision of their tumour is performed based on their pathological response to neoadjuvant treatment.

\section{Statistical Analysis}

Overall survival and disease specific survival estimates were analysed using the Kaplan-Meier method. Univariate and multivariate hazard ratios of independent predictors of survival were identified by Cox proportional hazard regression hazard analysis. The quality of TME, EMVI, pathological response to NaT, lymph node ratio and completeness of specimen excision were included into the model for multivariate 
analysis following statistical significance on univariate analysis. Where there is collinearity, variables were excluded from the multivariate model. Statistical significance was defined as $p<0.05$. All analyses were performed using STATA software version 14.0 (StataCorp 2015, TX).

\section{Ethics}

This was an assessment of clinical datasets and analysis was approved by the regional Caldicott guardian. 
$\underline{\text { Results }}$

A total of 878 patients were identified with primary rectal cancer in the study period, of whom, 596 patients had NaT for locally advanced rectal cancer and were included (Figure 1). The median age of the study population was 66 years (interquartile-range 57-73 years). The percentage of screen detected rectal cancers was $16.7 \%$. Complete pathological response was achieved in $19.5 \%$ of patients. The proportion of patients who had good partial, partial and minimal pathological response were 35.5\%, 33.4\% and $11.6 \%$ respectively. The majority $(87 \%)$ had an $\mathrm{RO}$ resection following $\mathrm{NaT}$. The quality of TME was good or complete in 453 patients (76\%). Table 1 displays the demographics of the study group by response to neoadjuvant therapy. There were no patients lost to follow up.

\section{Overall Survival}

The overall survival at the end of the study for minimal, partial, good partial and complete response is $37.3 \%, 39.0 \%, 58.9 \%$ and $75.6 \%$ respectively. The median follow-up period was 4.57 years (Interquartile range 2.21-8.15 years). The overall survival for patients with complete pathological response was $75.6 \%$ versus $37.3 \%$ for patients who had minimal response $(p<0.001)$. There is a significant difference in overall survival in the good partial versus partial response group (58.9\% vs $39 \%$, $p<0.001)$. There is no significant difference in the overall survival $(39.0 \%$ vs $37.3 \%, p=$ 0.186) for patients who had partial versus minimal response. Figure 2 shows the overall survival analysis by Kaplan Meier curves for response to NaT.

On univariate analysis, the pathological response to therapy is significantly associated with overall survival (table 2). There is a trend for an increasing hazards ratio associated with a poorer degree of pathological response. Patients with minimal pathological response have the poorest overall survival (Hazard ratio $5.30,95 \% \mathrm{Cl}$ 3.03-9.25, $p<0.05)$. There is an observable difference between the overall survival and in good partial and partial pathological response on the univariate analysis (Table 2). This difference is also evident on the Kaplan Meier Curve (Figure 2). Number of patients at risk of death during the follow-up are also shown under the Kaplan Meier Curve in figure 2. Patients with partial response showed no difference in overall 
survival when compared to minimal response to NaT. Other factors that elicited an effect on outcome were age, completeness of excision, stage, T-stage response, EMVI, Good TME, N-stage and lymph node ratio.

\section{Subgroup analysis}

The subgroup of 520 patients had complete excision of their tumour. A better degree of response was still associated with better overall and disease specific survival. [Overall survival: Good partial (Hazard ratio 1.95, 95\% Cl 1.15-3.29, p<0.05), partial (Hazard ratio 3.57, 95\% Cl 2.11-6.04, p<0.05), minimal (Hazard ratio 3.73, 95\% Cl 1.96-7.12,p<0.05) Disease specific survival: Good partial (Hazard ratio 3.88, 95\% Cl 1.50-10.06, p<0.05), partial (Hazard ratio 7.97, 95\% Cl 3.11-20.41, p<0.05), minimal (Hazard ratio 10.54, 95\% Cl 3.83-29.03,p<0.05)].

In the 76 patients who had incomplete excision, the mean survival is 2.4 years. There were 11 patients who had a good partial response and the survival was $36.4 \%$ at 5 years (4 survivors). In the group who had partial response, survival was $26.2 \%$ at 5 years (11 survivors). Lastly, 22 patients had minimal response, and the 5 years survival was $9.1 \%$ ( 2 survivors).

\section{Multivariate analysis of overall survival}

A multivariate analysis adjusting for pathological response, quality of TME, EMVI, lymph node ratio, completeness of excision and age was performed (Table 3). Pathological response, in particular, partial (HR 2.27, 95\% Cl 1.36 to 3.86, p<0.05) and minimal response $(\mathrm{HR} 2.79,95 \% \mathrm{Cl} 1.49$ to $5.21, \mathrm{p}<0.05)$ remained an independent variable for poorer survival. The hazards ratio demonstrated on our model appears to be similar in both partial and minimal response for overall survival. Patients with partial or minimal response are roughly twice as likely to succumb to their disease as compared to patients with good partial response. 
The disease specific survival for minimal, partial, good partial and complete response was $43.1 \%, 49.2 \%, 76.6 \%$ and $94.7 \%$ respectively. The disease-specific survival was $94.7 \%$ versus $43.1 \%$ for complete pathological response versus minimal response ( $p$ $<0.001)$. There is no significant difference in the disease specific survival $(49.2 \%$ vs $43.1 \%, p=0.092$ ) for patients who have partial versus minimal response. These data are shown in the supplementary figures.

Supplementary figure one shows disease specific survival analysis by Kaplan Meier curves for response to NaT respectively. On univariate analysis, the pathological response to therapy is significantly associated with disease-specific survival. Patients with minimal pathological response have the poorest disease-specific survival (Hazard ratio $15.38,95 \% \mathrm{Cl} 5.91-39.99, \mathrm{p}<0.05)$. There is an observable difference between the disease-specific survival and in good partial and partial pathological response on the univariate analysis. However, patients with partial response showed no difference in disease-specific survival when compared to minimal response to $\mathrm{NaT}$

On multivariate analysis of disease specific survival, the hazards ratio demonstrated on our model appears to be similar in both partial and minimal response for diseasespecific survival. The quality of TME, lymph node harvest ratio, completeness of excision and age were associated factors in disease specific survival $(p<0.05)$. 


\section{Discussion}

This study demonstrates the tumour regression after administration of $\mathrm{NaT}$ is an independent predictor of survival in locally advanced rectal cancer. The significance of $\mathrm{pCR}$ is well known to be of importance. Our study adds to the literature by demonstrating, a good partial response to $\mathrm{NaT}$ has a better outcome than partial or minimal response, even if an $\mathrm{R} 0$ resection is achieved. There was no difference in outcome between partial and minimal response groups.

The improvement in outcome of pCR has been demonstrated in another studies (17) and the minimal response outcome findings are in concordance with the observations demonstrated in other case series $(11)(12)(18)(9)$. A study analyzing the outcomes from the German rectal cancer trial has also demonstrated the correlation between degree of TRG with long term survival outcome (3)(19). Interestingly, our results have supported these works by showing that the survival is similar in patients who have had either partial or minimal pathological response. However, having a good partial pathological response, improves their prognosis significantly. This further advocates for a nuanced analysis of response to NaT to be reported regularly.

We postulate that the degree of pathological response could be considered as a factor for deciding which patients should or should not undergo adjuvant treatment or indeed whether a more intensive regimen could be warranted for a lesser degree of pathological response. Indeed, a recent study has reported using the TRG system to help in identifying patients who may benefit from adjuvant treatment (20). The authors have sub stratified pathological response (good versus poor) and concluded that good response to $\mathrm{NaT}$ is an independent predictor of survival in patients with stage 2 and 3 rectal cancer. We went a step further by stratifying the 'partials' into good partial or partial. However, we acknowledge that more studies are required before implementing such a change in decision making practice.

Recently, a new surrogate end point, the neoadjuvant rectal score (NAR) was developed to predict the long term outcomes following NaT (21). Even though NAR has been adopted in some clinical trials, its value as a surrogate endpoint for overall 
survival remains unclear (22). Therefore, an open mind should still be kept on what the optimal end point for predicting survival should be for rectal cancer.

It is of clinical importance if the degree of pathological response could be predicted pre-operatively. Of particular interest will be the prediction of a complete pathological response pre-operatively. We have demonstrated that this group of patients have excellent prognosis and surgery with its associated risks could potentially be an overtreatment. The 'watch and wait' strategy pioneered by Habr-Gama, is an alternative management strategy for patients with complete response $(23)(24)(25)$. It is an attractive option for patients and, for some, even an avoidance of surgery and colostomy after ensuring no oncological compromise (24).

We had an incomplete excision rate of $32 \%(22 / 69)$ in patients with a pathological minimal response and $21 \%$ (42/199) in patients with pathological partial response. This is in contrast to an incomplete excision rate of $5 \%$ for patients with pathological good partial response. We acknowledge the high $\mathrm{R} 1$ rates are high in the minimal response group and we have adopted an increased rate of pelvic exenteration to improve the negative resection margin. It is clear R0 resection for locally advanced primary rectal cancer is key to improved survival. Most recently, this has been demonstrated by the PelvEx international collaborative group (26).

The results of the magnetic resonance tumour regression grade (mrTRG) (TRIGGER trial) could potentially be very useful in stratifying between poor responders versus good responders (27). If the mrTRG demonstrates poor response different oncology options may be considered or beyond TME operative approach may be adopted.

There are a number of TRG systems with comparable results (9). Assessment of TRG is highly subjective dependent on the pathology expertise but the effort to sub stratify partial pathological response into good partial or partial (poor) may be of benefit to patients for the reasons stated above. Our analysis is benefitted by a consistent TRG reporting strategy overseen by an expert gastrointestinal pathology department with high quality reporting. The TRG reporting scale used in this study was developed and stratified by this pathology department. Our analysis (both univariate and multivariate) has shown that lymph node harvest ratio has an impact on survival. The presence of 
EMVI is associated with a slightly poorer survival however this did not achieve statistical significance in our adjusted multivariate model which could be due to the small sample size.

One of the strengths of our study is that we have consistently used the same TRG system from 2005 when we first collected our data. The TRG for all specimen have been assessed by expert gastrointestinal pathologists. The Royal College of Pathologists, UK previously used a 3 point TRG system for reporting of rectal cancer specimens but as of 2018 (version 4) has adopted a 4 point TRG system which is similar to the system that we have been using. Despite similarities in both TRG systems, further studies to validate our system against that adopted by the Royal College of Pathologist in a wider population is recommended.

Our study does have limitations including the inherent bias of a retrospective analysis of a prospectively maintained database. Our dataset does not capture whether a patient has developed local recurrences or metastatic disease individually. However, we have the summative endpoint of disease specific survival. In these analysis, we see the same findings reflected in the overall survival analysis.

\section{Conclusion}

In the presence of long-term follow-up, this study has demonstrated that the consistent use of a histopathological tumour regression grading system was an independent predictor of survival for patients undergoing neoadjuvant therapy for locally advanced rectal cancer. This may indicate that, with further data and analysis, tumour regression grade could be used clinically as a risk stratification tool for further adjuvant therapy in this group. 


\section{References}

1. Hong TS, Clark JW, Haigis KM. Cancers of the Colon and Rectum: Identical or Fraternal Twins? Cancer Discovery. 2012 Feb;2(2):117-21.

2. Kalyan A, Rozelle S, Benson A. Neoadjuvant treatment of rectal cancer: where are we now? Gastroenterol Rep (Oxf). 2016 Aug;4(3):206-9.

3. Sauer R, Becker H, Hohenberger W, Rödel C, Wittekind C, Fietkau R, et al. Preoperative versus Postoperative Chemoradiotherapy for Rectal Cancer. New England Journal of Medicine. 2004 Oct 21;351(17):1731-40.

4. Sebag-Montefiore D, Stephens RJ, Steele R, Monson J, Grieve R, Khanna S, et al. Preoperative radiotherapy versus selective postoperative chemoradiotherapy in patients with rectal cancer (MRC CR07 and NCIC-CTG C016): a multicentre, randomised trial. The Lancet. 2009 Mar;373(9666):811-20.

5. Lee Y-C, Lee Y-L, Chuang J-P, Lee J-C. Differences in Survival between Colon and Rectal Cancer from SEER Data. Goel A, editor. PLoS ONE. 2013 Nov 12;8(11):e78709.

6. Rombouts AJM, Hugen N, Verhoeven RHA, Elferink MAG, Poortmans PMP, Nagtegaal ID, et al. Tumor response after long interval comparing 5x5Gy radiation therapy with chemoradiation therapy in rectal cancer patients. European Journal of Surgical Oncology. 2018 Jul;44(7):1018-24.

7. Mandard AM, Dalibard F, Mandard JC, Marnay J, Henry-Amar M, Petiot JF, et al. Pathologic assessment of tumor regression after preoperative chemoradiotherapy of esophageal carcinoma. Clinicopathologic correlations. Cancer. 1994 Jun $1 ; 73(11): 2680-6$.

8. Kim SH, Chang HJ, Kim DY, Park JW, Baek JY, Kim SY, et al. What Is the Ideal Tumor Regression Grading System in Rectal Cancer Patients after Preoperative Chemoradiotherapy? Cancer Research and Treatment. 2016 Jul 15;48(3):9981009. 
9. Zhang L-N, Xiao W-W, Xi S-Y, OuYang P-Y, You K-Y, Zeng Z-F, et al. Pathological Assessment of the AJCC Tumor Regression Grading System After Preoperative Chemoradiotherapy for Chinese Locally Advanced Rectal Cancer: Medicine. 2016 Jan;95(3):e2272.

10. De-Torres-Olombrada MV, Juez-Martel I, Rodríguez-Caravaca G, DuranPoveda M. Role of the complete pathological response in rectal cancer: Value as a prognostic factor. JOURNAL OF SURGICAL ONCOLOGY. 2018 Jan 10;1(1):25.

11. Jalilian M, Davis S, Mohebbi M, Sugamaran B, Porter IW, Bell S, et al. Pathologic response to neoadjuvant treatment in locally advanced rectal cancer and impact on outcome. Journal of Gastrointestinal Oncology. 2016 Aug;7(4):6038.

12. Rödel C, Martus P, Papadoupolos T, Füzesi L, Klimpfinger M, Fietkau R, et al. Prognostic Significance of Tumor Regression After Preoperative Chemoradiotherapy for Rectal Cancer. Journal of Clinical Oncology. 2005 Dec;23(34):8688-96.

13. Bouzourene H, Bosman FT, Seelentag W, Matter M, Coucke P. Importance of tumor regression assessment in predicting the outcome in patients with locally advanced rectal carcinoma who are treated with preoperative radiotherapy. Cancer. 2002 Feb 15;94(4):1121-30.

14. Glynne-Jones R, Sebag-Montefiore D, McDonald A, Falk S, Maughan T. Preliminary phase II SOCRATES study results: Capecitabine (CAP) combined with oxaliplatin (OX) and preoperative radiation (RT) in patients (pts) with locally advanced rectal cancer (LARC). Journal of Clinical Oncology. 2004 Jul 15;22(14_suppl):3575-3575.

15. Brown GT, Cash B, Alnabulsi A, Samuel LM, Murray GI. The expression and prognostic significance of bcl-2-associated transcription factor 1 in rectal cancer following neoadjuvant therapy. Histopathology. 2016 Mar;68(4):556-66. 
16. Loughrey M, Quirke P, Shepherd N. Dataset for histopathological reporting of colorectal cancer. London: The Royal College of Pathologists; 2018 Sep.

17. Maas M, Nelemans PJ, Valentini V, Das P, Rödel C, Kuo L-J, et al. Long-term outcome in patients with a pathological complete response after chemoradiation for rectal cancer: a pooled analysis of individual patient data. The Lancet Oncology. 2010 Sep;11(9):835-44.

18. Mancini R, Pattaro G, Diodoro MG, Sperduti I, Garufi C, Stigliano V, et al. Tumor Regression Grade After Neoadjuvant Chemoradiation and Surgery for Low Rectal Cancer Evaluated by Multiple Correspondence Analysis: Ten Years as Minimum Follow-up. Clinical Colorectal Cancer. 2018 Mar;17(1):e13-9.

19. Fokas E, Liersch T, Fietkau R, Hohenberger W, Beissbarth T, Hess C, et al. Tumor Regression Grading After Preoperative Chemoradiotherapy for Locally Advanced Rectal Carcinoma Revisited: Updated Results of the CAO/ARO/AIO-94 Trial. JCO. 2014 May 20;32(15):1554-62.

20. Song C, Chung J-H, Kang S-B, Kim D-W, Oh H-K, Lee HS, et al. Impact of Tumor Regression Grade as a Major Prognostic Factor in Locally Advanced Rectal Cancer after Neoadjuvant Chemoradiotherapy: A Proposal for a Modified Staging System. Cancers. 2018 Sep 7;10(9):319.

21. George TJ, Allegra CJ, Yothers G. Neoadjuvant Rectal (NAR) Score: a New Surrogate Endpoint in Rectal Cancer Clinical Trials. Current Colorectal Cancer Reports. 2015 Oct;11(5):275-80.

22. van der Valk MJM, Vuijk FA, Putter $\mathrm{H}$, van de Velde $\mathrm{CJH}$, Beets GL, Hilling DE. Disqualification of Neoadjuvant Rectal Score Based on Data of 6596 Patients From the Netherlands Cancer Registry. Clinical Colorectal Cancer. 2019 Jun;18(2):e231-6.

23. Habr-Gama A, Perez RO, Nadalin W, Sabbaga J, Ribeiro U, Silva e Sousa AH, et al. Operative versus nonoperative treatment for stage 0 distal rectal cancer following chemoradiation therapy: long-term results. Ann Surg. 2004 Oct;240(4):711-7; discussion 717-718. 
24. Renehan AG, Malcomson L, Emsley R, Gollins S, Maw A, Myint AS, et al. Watch-and-wait approach versus surgical resection after chemoradiotherapy for patients with rectal cancer (the OnCoRe project): a propensity-score matched cohort analysis. Lancet Oncol. 2016 Feb;17(2):174-83.

25. Dossa F, Chesney TR, Acuna SA, Baxter NN. A watch-and-wait approach for locally advanced rectal cancer after a clinical complete response following neoadjuvant chemoradiation: a systematic review and meta-analysis. Lancet Gastroenterol Hepatol. 2017 Jul;2(7):501-13.

26. The PelvEx Collaborative. Surgical and Survival Outcomes Following Pelvic Exenteration for Locally Advanced Primary Rectal Cancer: Results From an International Collaboration. Annals of Surgery. 2019 Feb;269(2):315-21.

27. Battersby NJ, Dattani M, Rao S, Cunningham D, Tait D, Adams R, et al. A rectal cancer feasibility study with an embedded phase III trial design assessing magnetic resonance tumour regression grade (mrTRG) as a novel biomarker to stratify management by good and poor response to chemoradiotherapy (TRIGGER): study protocol for a randomised controlled trial. Trials [Internet]. 2017 Dec [cited 2018 Apr 10];18(1). Available from: http://trialsjournal.biomedcentral.com/articles/10.1186/s13063-017-2085-2 


\begin{tabular}{|c|c|c|c|c|c|}
\hline & Total & $\begin{array}{l}\text { Minimal } \\
\text { response }\end{array}$ & $\begin{array}{l}\text { Partial } \\
\text { Response }\end{array}$ & $\begin{array}{l}\text { Good Partial } \\
\text { Response }\end{array}$ & $\begin{array}{l}\text { Complete } \\
\text { Response }\end{array}$ \\
\hline Number of patients & 596 & $69(11.6 \%)$ & $199(33.4 \%)$ & $212(35.6 \%)$ & $\begin{array}{l}116 \\
(19.4 \%)\end{array}$ \\
\hline Median age (years) & 66 & 68 & 66 & 66 & 64 \\
\hline Male:Female & $373: 223$ & $49: 20$ & $118: 81$ & $133: 79$ & $73: 43$ \\
\hline Screen Detected & 89 & $10(11.2 \%)$ & $\begin{array}{l}24 \\
(27 \%)\end{array}$ & $37(41.6 \%)$ & $18(20.2 \%)$ \\
\hline \multicolumn{6}{|l|}{ yPT stage } \\
\hline $\begin{array}{l}\text { T0 } \\
\text { T1 } \\
\text { T2 } \\
\text { T3 } \\
\text { T4 } \\
\end{array}$ & $\begin{array}{l}126 \\
56 \\
127 \\
268 \\
19 \\
\end{array}$ & $\begin{array}{l}0(0.0 \%) \\
3(5.4 \%) \\
11(8.7 \%) \\
50(18.7 \%) \\
5(26.3 \%)\end{array}$ & $\begin{array}{l}0(0.0 \%) \\
8(14.3 \%) \\
33(26 \%) \\
146(54.5 \%) \\
11(57.9 \%)\end{array}$ & $\begin{array}{l}0(0.0 \%) \\
45(80.3 \%) \\
83(65.3 \%) \\
71(26.8 \%) \\
3(15.8 \%)\end{array}$ & $\begin{array}{l}126(100 \%) \\
0(0.0 \%) \\
0(0.0 \%) \\
0(0.0 \%) \\
0(0.0 \%)\end{array}$ \\
\hline \multicolumn{6}{|l|}{ yPN stage } \\
\hline $\begin{array}{l}\mathrm{N} 0 \\
\mathrm{~N} 1 \\
\mathrm{~N} 2\end{array}$ & $\begin{array}{l}455 \\
102 \\
39\end{array}$ & $\begin{array}{l}47(10.3 \%) \\
11(10.8 \%) \\
11(28.2 \%)\end{array}$ & $\begin{array}{l}118(25.9 \%) \\
60(58.8 \%) \\
21(53.8 \%) \\
\end{array}$ & $\begin{array}{l}175(38.5 \%) \\
30(30.4 \%) \\
7(18 \%)\end{array}$ & $\begin{array}{l}116(25.3 \%) \\
0(0.0 \%) \\
0(0.0 \%)\end{array}$ \\
\hline \multicolumn{6}{|l|}{$\begin{array}{l}\text { Extramural venous } \\
\text { invasion }\end{array}$} \\
\hline $\begin{array}{l}\text { Yes } \\
\text { No } \\
\end{array}$ & $\begin{array}{l}102 \\
494 \\
\end{array}$ & $\begin{array}{l}32(31.4 \%) \\
37(7.5 \%) \\
\end{array}$ & $\begin{array}{l}55(53.9 \%) \\
144(29.1 \%) \\
\end{array}$ & $\begin{array}{l}14(13.7 \%) \\
198(40.1 \%) \\
\end{array}$ & $\begin{array}{l}0(0.0 \%) \\
116(23.3 \%) \\
\end{array}$ \\
\hline \multicolumn{6}{|l|}{ Complete Excision } \\
\hline $\begin{array}{l}\text { Yes } \\
\text { No }\end{array}$ & $\begin{array}{l}520 \\
76 \\
\end{array}$ & $\begin{array}{l}47(9 \%) \\
22(28.9 \%)\end{array}$ & $\begin{array}{l}157(30.2 \%) \\
42(55.3 \%) \\
\end{array}$ & $\begin{array}{l}201(38.7 \%) \\
11(15.8 \%) \\
\end{array}$ & $\begin{array}{l}116(22.1 \%) \\
0(0.0 \%)\end{array}$ \\
\hline $\begin{array}{l}\text { Lymph Nodes } \\
\text { examined (Median) }\end{array}$ & 17 & 18 & 18 & 17 & 15 \\
\hline $\begin{array}{l}\text { Chemotherapy only } \\
\text { Radiotherapy only } \\
\text { Chemoradiotherapy }\end{array}$ & $\begin{array}{l}58 \\
114 \\
424\end{array}$ & $\begin{array}{l}21(36.2 \%) \\
14(12.3 \%) \\
34(8.0 \%)\end{array}$ & $\begin{array}{l}20(34.5 \%) \\
36(31.6 \%) \\
143(33.7 \%)\end{array}$ & $\begin{array}{l}17(29.3 \%) \\
48(42.1 \%) \\
147(34.7 \%)\end{array}$ & $\begin{array}{l}0(0 \%) \\
16(14 \%) \\
100(23.6 \%)\end{array}$ \\
\hline
\end{tabular}

Table 1: Demographics and pathological characteristics of patients who had neoadjuvant therapy 


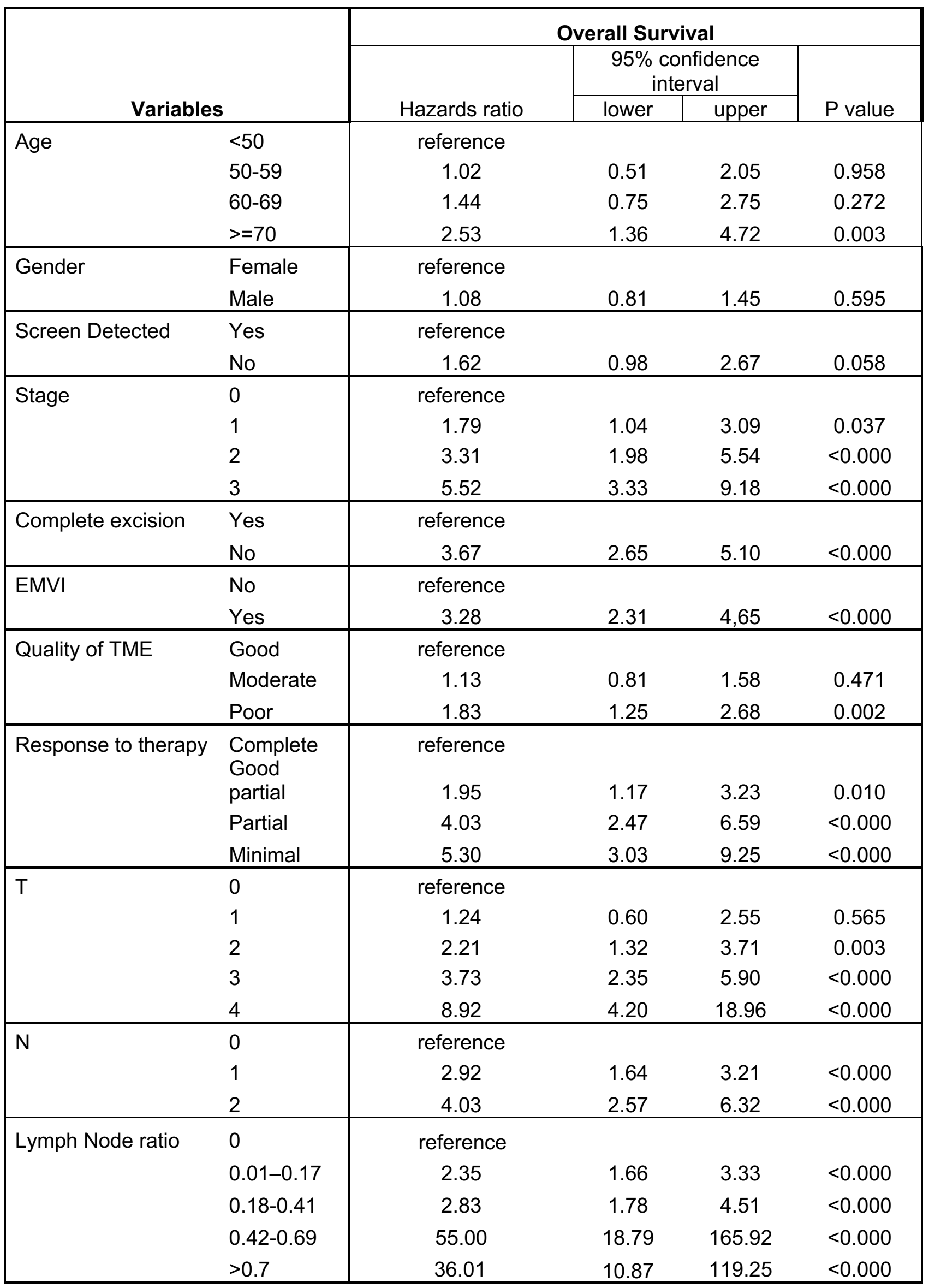

Table 2 - Univariate analysis of variables associated with overall survival 


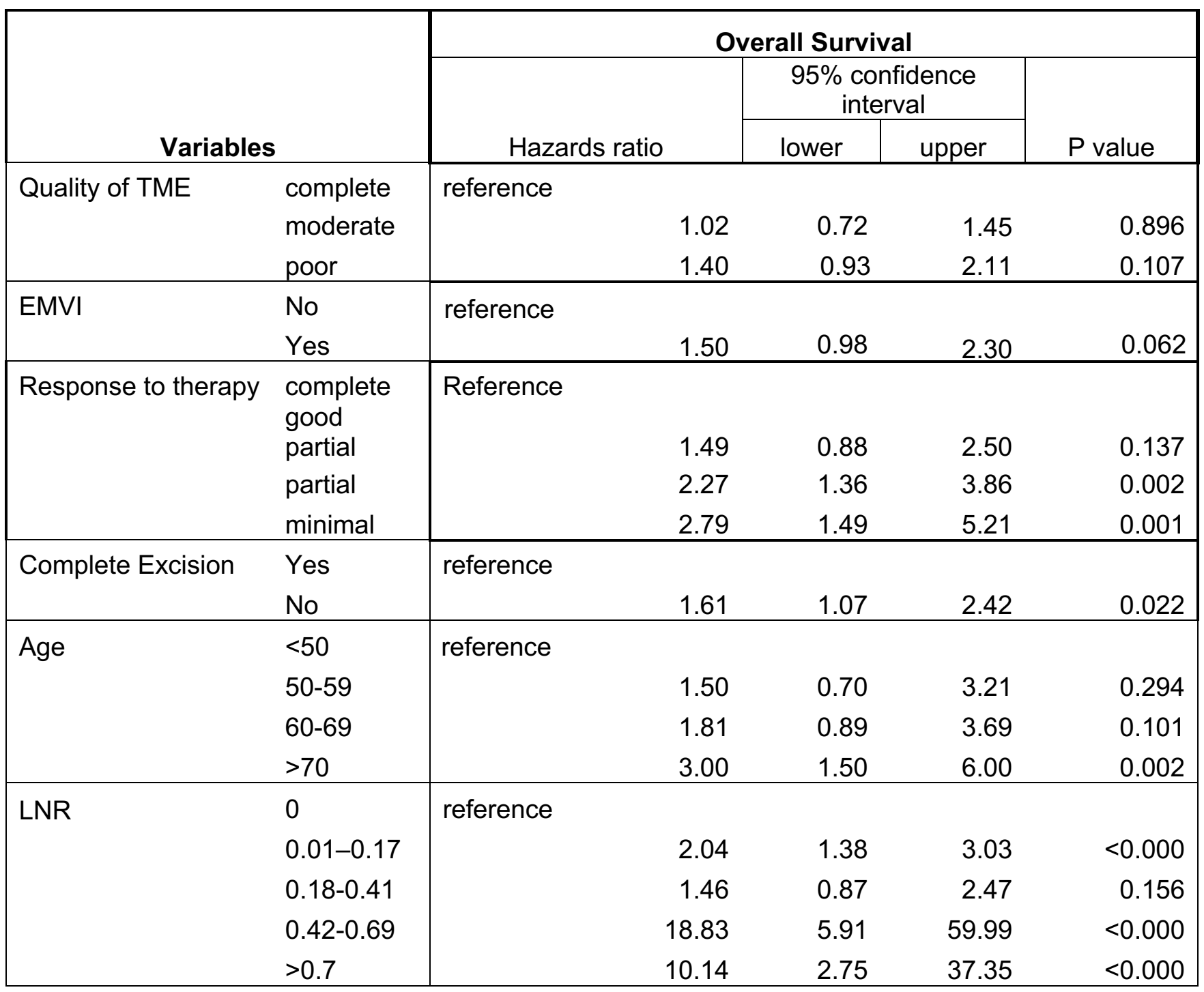

Table 3 - Multivariate analysis of clinical variables associated with overall survival 